\title{
The prognostic value of histological grading of posterior fossa ependymomas in children: a Children's Oncology Group study and a review of prognostic factors
}

\author{
Tarik Tihan ${ }^{1}$, Tianni Zhou ${ }^{2}$, Emi Holmes², Peter C Burger ${ }^{3}$, Sema Ozuysal ${ }^{4}$ and \\ Elisabeth J Rushing ${ }^{5}$ \\ ${ }^{1}$ Department of Pathology, University of California, San Francisco, CA, USA; ${ }^{2}$ Department of Pathology, \\ Johns Hopkins University, Baltimore, MD, USA; ${ }^{3}$ COG Statistical Office, Arcadia, CA, USA; ${ }^{4}$ Department of \\ Pathology, Uludag University School of Medicine, Bursa, Turkey and ${ }^{5}$ Department of Neuropathology \\ and Ophthalmic Pathology, Armed Forces Institute of Pathology, Washington, DC, USA
}

\begin{abstract}
We performed a retrospective analysis of 96 pediatric posterior fossa ependymomas in order to determine the prognostic value of histological grade based on the current WHO grading scheme. The patients were selected among Children's Oncology Group (previously Pediatric Oncology Group-POG) patients enrolled in clinical trials, and on the basis of central pathology review, location, and age. We excluded entities such as subependymoma, myxopapillary, or clear-cell ependymoma, after a consensus diagnosis by three neuropathologists. A total of 66 males and 30 females with a median age of 48 months were identified. The group was analyzed to determine the effects of histological grade, age, gender, and extent of resection on event-free and overall survival. Our results showed that extent of resection, age, and histological grade were independent prognostic variables for event-free survival. The relative risk for extent of resection and histological grade was calculated as $3.59(P<0.001)$ and $3.58(P<0.001)$, respectively. Overall survival significantly correlated with extent of resection and age, but not with histological grade. We compared our results with peer-reviewed publications on pediatric intracranial ependymomas in the English language between 1990 and 2005. Selection criteria identified 32 manuscripts involving 1444 patients. Extent of resection was a significant factor in 21, age in 12, and histological grading in nine of these studies. Other factors reported to be significant by more than one study included tumor location and radiation treatment. Our findings suggest that histological grade (WHO Grade II vs III) is an independent prognostic indicator for event-free survival, but may not be so for overall survival in pediatric posterior fossa ependymomas. We believe that an accurate assessment of the prognostic value of histological grade depends on the selection of a well-characterized clinical cohort of sufficient size, and the inclusion of relevant histological criteria as outlined in the WHO classification scheme.
\end{abstract}

Modern Pathology (2008) 21, 165-177; doi:10.1038/modpathol.3800999; published online 14 December 2007

Keywords: ependymoma; childhood brain tumor; posterior fossa; review pediatric glioma; prognosis

Ependymal tumors are among the most common pediatric tumors in the posterior fossa. The typical ependymoma occurs in association with the fourth ventricle, but can be seen throughout the neuraxis. Histologically, ependymomas are graded in a twotiered scheme (Grade II or III) in the current WHO classification, while variants such as myxopapillary

Correspondence: Dr T Tihan, MD, PhD, UCSF Medical Center, Department of Pathology, Neuropathology Unit, Room M551, 505 Parnassus Avenue, San Francisco, CA 94143-0102, USA.

E-mail: tarik.tihan@ucsf.edu

Received 10 May 2007; revised 24 September 2007; accepted 1 October 2007; published online 14 December 2007 ependymoma or sub-ependymoma are considered Grade I lesions. ${ }^{1}$

The management of pediatric ependymomas is one of the most controversial topics in pediatric neuro-oncology and may differ significantly among institutions. ${ }^{2}$ This controversy is partly related to uncertainties about the patient and tumor characteristics that are predictive of behavior. Much information has been provided from single-institution and consortia studies, but the prognostic relevance of most factors are still being debated. ${ }^{2}$ In some studies, the value of histological grading has been undermined by difficulties in correlating this parameter with clinical outcome. ${ }^{3}$ This conclusion was not uniformly reached in all studies. ${ }^{4}$ Thus, it is 
critical to determine if, and to what extent, grading influences prognosis.

The difficulty in grading posterior fossa ependymomas may be partly attributed to inter-observer variability, variations in definitions, and the use of different histological criteria. While the majority of studies reported the use of the WHO scheme, others have opted to employ different schemes. In other instances, some studies merely correlated individual microscopic features without providing a histological grade. An example of this practice is the interpretation of necrosis, which is variably present in the majority of ependymomas. Necrosis has been both considered and ignored as a grading criterion. When considered among the criteria, some studies did find that necrosis was a prognostic variable ${ }^{5}$ while others have not found a correlation. ${ }^{6}$ One study reported a positive correlation with necrosis and poor outcome, but not with anaplastic histology, (ie, histological grade). ${ }^{7}$

There may be additional factors not related with pathological interpretation that can complicate histological grading. One such issue is the tissue sample size, and whether it is representative of the whole tumor. Small biopsies of tumors could be graded differently given additional tissue. Patient selection can further complicate statistical analysis and alter conclusions. For example, inclusion of adults and/or patients with other tumor categories such as ependymoblastoma is potentially problematic, leading one to overlook factors that may adversely influence the results. While such heterogeneous groups can still be analyzed using appropriate statistical methods, they are likely to fall short of being predictive. Furthermore, these confounding factors are not always appropriately considered in multivariate analyses, and sometimes not considered at all. Many studies may fall victim to small sample size, in which case the data may not allow the researchers to predict the significance of 'absence of correlation' with sufficient power.

We have undertaken this study to provide a critical review of histological grading of pediatric posterior fossa ependymomas, and to compare the literature findings with our own analysis of Children's Oncology Group (previously Pediatric Oncology Group-POG) patients. We postulated that such a comprehensive review could provide a more realistic interpretation of the problems in histological grading and help neuro-oncologists in their evaluation of risk factors for pediatric posterior fossa ependymomas.

\section{Materials and methods}

\section{Patients}

We identified all patients who were enrolled in the POG protocols between 1990 and 2000. The search included protocols 9060, 9132, 91235, 9233, 9237, 9330, and 9432, which represented nearly all ependymomas registered during this period. Appropriate permissions were obtained from institutional review boards of participating institutions. Patients without sufficient pathology material, clinical or follow-up information were excluded. Pathology material was in the form of $\mathrm{H} \& \mathrm{E}$ and unstained slides that had been submitted for central review. All available slides were reviewed by three neuropathologists independently, followed by a consensus review, and histological features were recorded. Immunohistochemical analyses using standard commercial antibodies were performed (when necessary and when slides were available) to confirm the diagnoses. All immunohistochemical analyses were performed at the Immunohistochemistry Laboratory of Johns Hopkins Pathology Department, and included appropriate positive and negative control tissue as recommended by the manufacturers.

Demographic data, dates of events, clinical and outcome information were obtained from the POG submission forms and the Statistical Office. Extent of resection was determined by review of the operative notes, and the neurosurgeons impression, when available.

\section{Pathological Review}

Pathological reviews were conducted without knowledge of the tumor location, patient age or other demographic features, and before the exclusion of patients with supratentorial ependymomas or other pathological entities. Pathological review considered a set of subjective criteria (Table 1) that were based on a review of literature and the current WHO Classification. ${ }^{8}$ Consensus was defined as agreement on the type and grade of neoplasm by all three participating neuropathologists, and all other cases were considered discrepant. The criteria included cut-off values for mitoses that

Table 1 Histological criteria used in the grading of posterior fossa ependymomas

\footnotetext{
Grade II ependymoma (all of the features below)

1 Perivascular pseudorosettes

2 Uniform nuclear morphology (focal nuclear pleomorphism allowed)

3 Mitoses less than five per 10 high-power field (HPF) (focal areas with 5-10 per $10 \mathrm{HPF}$ allowed)

$4 \quad$ No vascular endothelial proliferation (vascular proliferation associated with degenerative or cystic change or granulation-tissue type neovascularization allowed)

Grade III ependymoma (at least two of the features below)

1 Bona fide vascular endothelial proliferation with endothelial layering

2 Mitotic rate greater than 10 per $10 \mathrm{HPF}$ $\left(\times 400-0.238 \mathrm{~mm}^{2}\right)$ Palisading necrosis

$4 \quad$ Marked hypercellularity with nuclear pleomorphism and/or hyperchromasia
} 
were based on previous published reports and the authors' experience. The cut-off value for mitoses was chosen as $>10$ per 10 high-power fields $\left(0.238 \mathrm{~mm}^{2}\right)$ for Grade III ependymomas, and $<5$ for Grade II tumors. Mitotic rates between five and 10 were considered indeterminate and not sufficient to alter WHO grading assigned otherwise.

A joint review was conducted to record histological features that included presence of diffuse hyperchromasia and nuclear pleomorphism, foci of hypercellular nodules, number of mitoses $(<5$, $5-10$, and $>10$ ), presence of bona fide vascular endothelial proliferation, vascular proliferation associated with cystic change, necrosis (yes/no) as well as palisading necrosis recorded separately from the former.

\section{Statistical Analysis}

Analyses of clinical and outcome parameters with histopathological findings and tumor grade were performed by the COG statistical office and differences among groups separated according to histological grade were recorded (Grade II = standard risk vs Grade III = high risk). We compared the original diagnoses, individual diagnoses of each neuropathologist, and the consensus of all three neuropathologists in terms of event-free (EFS) and overall survival (OS). A separate analysis was conducted to determine the prognostic value of hypercellular nodules in tumors. Modified grading criteria for this purpose included those listed in Table 1, with 'focal hypercellular nodules with mitotic figures' as an additional criterion for Grade III ependymomas.

The primary end points for statistical analyses were OS, which was defined as the time from study entry to death from any cause, and EFS, which was defined as the minimum time from study entry to disease progression, disease relapse (as identified clinically), second malignancy or death from any cause. Non-parametric estimates of EFS and OS probabilities were obtained using the product limit (Kaplan-Meier) estimate, with standard errors computed using the Greenwood formula. The log-rank test was used in univariate analysis of differences in EFS and OS among patient subgroups. Cox regression methods were used for multivariate analysis. All $P$-values quoted are two-sided.

\section{Review of the Literature Data}

We performed a search of scientific peer-reviewed publications in the English literature that contained specific information on the prognostic factors in pediatric posterior fossa ependymomas published between 1990 and 2005 (also see Table 2). The search included all relevant keywords on PubMed and UCSF Library databases on three different occasions. All papers identified as relevant to the topic were screened, and the following criteria were used:

Inclusion criteria: (a) Studies on intracranial ependymomas with insignificant number of other entities; (b) studies exclusively on pediatric population (younger than 20 years) or those that provided a specific analysis of the pediatric population and (c) studies conducted between 1990 and 2005.

Exclusion criteria: (a) Studies that included adult patients ( $>20$ years); (b) studies with $25 \%$ or more tumors with other diagnoses including ependymoblastoma, subependymoma or myxopapillary ependymoma; (c) studies without pathological evaluation/grading and (d) case studies.

All studies were analyzed for statistical power using the available data and the pertinent findings of each manuscript were recorded.

\section{Results}

\section{Patients}

Among a total of 239 patients with ependymal tumors enrolled in the studies during 1990 and 2000, we excluded tumors without sufficient pathological material (biopsies) or insufficient clinical data. The remaining 115 patients were included in the pathological review by all neuropathologists without knowledge of the clinical information. Tumors in patients older than 15 years, supratentorial or spinal tumors, or consensus diagnoses other than classical Grade II and III ependymomas (ie, when all three neuropathologist agreed that the tumor did not belong to an ependymal category) were excluded after all neuropathologists reviewed all slides independently. After the selection process, 96 patients with posterior fossa ependymomas were included in the analysis. These patients had been enrolled in studies 9060, 9132, 9135, 9233, 9237, 9330, 9332, and 9432. Gender distribution, age, and extent of resection information are presented in Table 3. The median age of 96 patients was 48 months (range 1-184). There were 51 patients younger than 3 years $(53 \%)$. Extent of resection was recorded as gross total at the time of surgery in $49 \%$ of cases, and in all other cases the extent of resection was considered less than gross total. According to original institutional diagnoses, there were 81 Grade II, 11 Grade III ependymomas, and four tumors were given other diagnoses.

The diagnoses given by all three neuropathologists are presented in Table 4 . The rate of agreement among all three neuropathologists was $76 \%$. Pathology review was concordant (all three neuropathologists agree) in 70 patients (50 Grade II and 20 Grade III ependymomas). The consensus cases were the primary group in which the survival analyses were conducted. 
Table 2 Pediatric ependymomas-review of literature for prognostic value of histological grading

\begin{tabular}{|c|c|c|c|c|c|c|c|c|c|c|c|c|c|c|c|c|c|c|}
\hline \multirow[t]{2}{*}{ First author } & \multirow[t]{2}{*}{ Year } & \multirow{2}{*}{$\begin{array}{c}\text { Years } \\
\text { covered }\end{array}$} & \multicolumn{3}{|c|}{$N$} & \multirow{2}{*}{$\begin{array}{c}\text { Sex } \\
(M / F)\end{array}$} & \multicolumn{3}{|c|}{ Location } & \multicolumn{3}{|c|}{ Age at diagnosis (years) } & \multicolumn{3}{|c|}{ Histological grade } & \multicolumn{3}{|c|}{ Extent of surgery } \\
\hline & & & Total & $<15$ years & $>15$ years & & $P F$ & $S T$ & Spine & Median & Mean & Range & Grade II & Grade III & Other & TR-NTR & STR & $B x$ \\
\hline Goldwein ${ }^{9}$ & 1990 & 1970-1988 & 51 & 49 & 2 & 1.6 & 33 & 18 & 0 & NR & 4.5 & $0.4-20$ & 38 & 13 & 0 & 15 & 36 & 0 \\
\hline Nazar $^{\mathrm{a} 10}$ & 1990 & $1970-1987$ & 35 & 35 & 0 & 1.1 & 35 & 0 & 0 & NR & 5.9 & $0.2-16$ & 0 & 0 & $35^{\mathrm{a}}$ & 10 & 25 & 0 \\
\hline Sutton $^{11}$ & 1990 & 1975-1989 & 45 & 42 & 3 & NR & 30 & 14 & 0 & NR & 5.9 & $0.4-20$ & 35 & 10 & 0 & 23 & 18 & 0 \\
\hline Ernestus $^{12}$ & 1991 & 1951-1989 & 29 & 29 & 0 & NR & 0 & 29 & 0 & NR & NR & $0-10$ & 11 & 18 & 0 & 19 & 10 & 0 \\
\hline Chiu $^{13}$ & 1992 & $1955-1986$ & 25 & 25 & 0 & 1.1 & 16 & 9 & 0 & 2.5 & NR & $0-15$ & 11 & 14 & 0 & 5 & 20 & 0 \\
\hline Palma ${ }^{14}$ & 1993 & $1957-1986$ & 20 & 14 & 6 & 0.8 & 0 & 20 & 0 & NR & 12.5 & $1-20$ & 12 & 6 & 2 & 18 & 2 & 0 \\
\hline Rousseau $^{15}$ & 1994 & 1975-1989 & 80 & 80 & 0 & 1.3 & 63 & 17 & 0 & 3 & NR & $0.3-15.8$ & 54 & 18 & 8 & 38 & 38 & 4 \\
\hline Jayawickreme $^{16}$ & 1995 & 1979-1988 & 24 & NR & NR & 1.0 & 17 & 7 & 0 & NR & 4.5 & NR & 20 & 2 & 0 & 13 & 8 & 3 \\
\hline Pollack $^{7}$ & 1995 & 1975-1993 & 37 & NR & NR & NR & 25 & 12 & 0 & NR & NR & NR & NR & NR & NR & 23 & 14 & 0 \\
\hline Ernestus $^{17}$ & 1996 & 1951-1990 & 67 & 67 & 0 & NR & 28 & 39 & 0 & NR & NR & $0.1-15$ & 38 & 28 & 1 & 33 & 34 & 0 \\
\hline Foreman $^{6}$ & 1996 & 1976-1993 & 31 & 31 & 0 & 0.7 & 20 & 11 & 0 & 2 & 5.75 & $0.4-14$ & 21 & 10 & 0 & 7 & 23 & 1 \\
\hline Gerszten $^{5}$ & 1996 & $1954-1999$ & 66 & 37 & 0 & NR & 47 & 19 & 0 & NR & 5 & $0.5-14$ & NR & NR & NR & NR & NR & NR \\
\hline Merchant $^{18}$ & 1997 & 1978-1994 & 28 & 28 & 0 & 1.8 & 14 & 14 & 0 & 7 & NR & $2-20$ & 0 & 28 & 0 & 11 & 17 & 0 \\
\hline Perilongo $^{20}$ & 1997 & $1977-1993$ & 92 & 92 & 0 & 1.3 & 60 & 32 & 0 & 5.3 & NR & $0-13.5$ & 61 & 31 & 0 & 53 & 35 & 0 \\
\hline Needle $^{19}$ & 1997 & 1990-1992 & 19 & 19 & 0 & 1.7 & 11 & 8 & 0 & 7.5 & NR & $3-14$ & 10 & 9 & 0 & 9 & 10 & 0 \\
\hline Bennetto $^{21}$ & 1998 & 1976-1994 & 74 & 74 & 0 & 1.2 & 74 & 0 & 0 & 3 & NR & $0.4-16$ & NR & NR & NR & 28 & 46 & 0 \\
\hline Duffner $^{22}$ & 1998 & 1986-1990 & 48 & 48 & 0 & NR & NR & NR & NR & 1.5 & NR & $0-3$ & 34 & 14 & 0 & 19 & 28 & 1 \\
\hline Fouladi $^{23}$ & 1998 & 1988-1997 & 11 & 11 & 0 & 1.2 & 10 & 1 & 0 & 3.4 & NR & $1.2-11.5$ & 11 & 0 & 0 & 5 & 6 & 0 \\
\hline Robertson ${ }^{3}$ & 1998 & 1986-1992 & 32 & 29 & 3 & 1.1 & 21 & 11 & 0 & 7 & NR & $2-17.3$ & 20 & 12 & 0 & 26 & 5 & 1 \\
\hline Sala $^{24}$ & 1998 & 1976-1996 & 35 & 35 & 0 & 1.3 & 21 & 14 & 0 & NR & NR & $0.1-15$ & 22 & 13 & 0 & 19 & 16 & 0 \\
\hline Horn $^{25}$ & 1999 & $1987-1991$ & 83 & NR & NR & 1.5 & 62 & 19 & 2 & 4.3 & NR & $0.7-20$ & 51 & 31 & 1 & 57 & 25 & 1 \\
\hline Figarella-Branger ${ }^{4}$ & 2000 & 1976-1999 & 37 & 37 & 0 & 1.3 & 25 & 12 & 0 & 4 & NR & $0-16$ & 24 & 13 & 0 & 15 & 22 & 0 \\
\hline Grill $^{26}$ & 2001 & 1990-1998 & 73 & 73 & 0 & 1.2 & 60 & 13 & 0 & 2.3 & NR & $0-5$ & 8 & 60 & 5 & 62 & 11 & 0 \\
\hline Helseth $^{27}$ & 2001 & 1970-1998 & 25 & 23 & 2 & 1.3 & 18 & 2 & 5 & NR & 5.6 & $1-18.7$ & NR & NR & NR & 18 & 7 & 0 \\
\hline Vinchon $^{28}$ & 2001 & 1985-1999 & 18 & 18 & 0 & 0.8 & 0 & 18 & 0 & NR & 9.2 & $0.25-15$ & 4 & 14 & 0 & 13 & 4 & 1 \\
\hline Dyer $^{29}$ & 2002 & NR & 42 & 42 & 0 & 0.7 & 35 & 7 & 0 & 4.3 & 5.3 & $1.2-13$ & 18 & 24 & 0 & 16 & 26 & 0 \\
\hline Merchant $^{30}$ & 2002 & 1982-1999 & 50 & NR & NR & 0.9 & 38 & 12 & 0 & 2.8 & NR & $0.5-17.8$ & 36 & 14 & 0 & 41 & 9 & 0 \\
\hline van Veelen ${ }^{31}$ & 2002 & 1980-1999 & 83 & 83 & 0 & 1.2 & 65 & 18 & 0 & NR & 3.0 & $0-16$ & 19 & 62 & 2 & 60 & 21 & 2 \\
\hline Zamecnik $^{32}$ & 2003 & 1985-1995 & 31 & 31 & 0 & 1.6 & 15 & 16 & 0 & NR & 5.8 & $1.0-14$ & 11 & 20 & 0 & 9 & 22 & 0 \\
\hline Jaing $^{33}$ & 2004 & 1985-2002 & 43 & NR & NR & 1.6 & 28 & 15 & 0 & 6.6 & NR & $0.7-18$ & 20 & 23 & 0 & 18 & 19 & 6 \\
\hline Agaoglu ${ }^{34}$ & 2005 & 1989-2001 & 40 & 40 & 0 & 1.2 & 18 & 18 & 2 & 5.5 & NR & $0.1-15$ & 18 & 22 & 0 & 20 & 18 & 2 \\
\hline Vinchon $^{35}$ & 2005 & 1979-2003 & 70 & NR & NR & 1.7 & 50 & 20 & 0 & NR & NR & $0-15$ & 31 & 34 & 5 & 38 & 29 & 3 \\
\hline
\end{tabular}

Bx, biopsy; NR, not reported.

Highlighted text identifies studies that found histological grade as a significant independent variable; PF, posterior fossa; ST, supratentorial; STR, subtotal resection; TR-NTR, total or near-total resection.

${ }^{\mathrm{a}}$ The study by Nazar et al used grading criteria different from the WHO scheme. 


\section{Histological Features}

Histologically, all tumors demonstrated evidence of ependymal differentiation, with Grade II neoplasms showing perivascular pseudorosettes (Figure 1a and b), and some with occasional true ependymal rosettes (Figure 1c) and occasionally undermining a single layer of well-differentiated ependymal cells.

Table 3 Patient characteristics and univariate analysis of EFS and OS in 96 posterior fossa ependymomas

\begin{tabular}{|c|c|c|c|c|c|}
\hline \multirow[t]{2}{*}{ Variable } & \multirow[t]{2}{*}{ No. } & \multicolumn{2}{|c|}{ Overall survival } & \multicolumn{2}{|c|}{ Event-free survival } \\
\hline & & $\begin{array}{c}\text { 5-year } \\
\text { OS }\end{array}$ & Log-rank $P$ & $\begin{array}{c}\text { 5-year } \\
\text { EFS }\end{array}$ & Log-rank $\mathrm{P}$ \\
\hline \multicolumn{6}{|l|}{ Age } \\
\hline$<3$ years & 51 & $30 \pm 6.5$ & 0.019 & $16 \pm 5.2$ & 0.004 \\
\hline$>3$ years & 45 & $52 \pm 7.6$ & & $48 \pm 7.5$ & \\
\hline \multicolumn{6}{|l|}{ Sex } \\
\hline Male & 66 & $38 \pm 6.1$ & 0.44 & $30 \pm 5.8$ & 0.57 \\
\hline Female & 30 & $46 \pm 9.1$ & & $33 \pm 8.6$ & \\
\hline \multicolumn{6}{|c|}{ Extent of resection } \\
\hline Gross total & 49 & $58 \pm 7.1$ & 0.002 & $46 \pm 7.2$ & 0.0005 \\
\hline$<$ Total & 47 & $22 \pm 6.1$ & & $15 \pm 5.3$ & \\
\hline \multicolumn{6}{|l|}{ Pathologist 1} \\
\hline Grade II & 56 & $46 \pm 6.8$ & 0.14 & $40 \pm 6.7$ & 0.017 \\
\hline Grade III & 40 & $32 \pm 7.4$ & & $20 \pm 6.3$ & \\
\hline \multicolumn{6}{|l|}{ Pathologist 2} \\
\hline Grade II & 68 & $43 \pm 6.1$ & 0.19 & $38 \pm 5.9$ & 0.003 \\
\hline Grade III & 20 & $30 \pm 10$ & & $10 \pm 6.7$ & \\
\hline \multicolumn{6}{|l|}{ Pathologist 3} \\
\hline Grade II & 68 & $44 \pm 6.1$ & 0.18 & $38 \pm 6.0$ & 0.003 \\
\hline Grade III & 20 & $30 \pm 10$ & & $10 \pm 6.7$ & \\
\hline \multicolumn{6}{|c|}{ Consensus review (all agree) } \\
\hline Grade II & 50 & $45 \pm 7.1$ & 0.16 & $39 \pm 7.0$ & 0.003 \\
\hline Grade III & 20 & $30 \pm 10$ & & $10 \pm 6.7$ & \\
\hline \multicolumn{6}{|c|}{ Modified grading with additional criterion $^{\mathrm{a}}$} \\
\hline Grade II & 60 & $43 \pm 6.5$ & 0.38 & $37 \pm 6.3$ & 0.076 \\
\hline Grade III & 33 & $36 \pm 8.4$ & & $24 \pm 7.5$ & \\
\hline
\end{tabular}

EFS, event-free survival; OS, overall survival.

${ }^{\mathrm{a}}$ Focal hypercellular nodules were used as an additional criterion to increase the WHO grade.

Statistically significant numbers are presented in bold.
Rare foci of few bizarre cells with hyperchromatic nuclei were encountered, but this was always a very small component (Figure 1d). Foci of necrosis without palisading were encountered in the majority of the tumors (Figure 1e). Whenever available, immunohistochemical studies revealed variable GFAP positivity that was accentuated in the perivascular regions (Figure 1f). In those cases in which neuronal stains were available, synaptophysin, chromogranin or Neu-N was negative in tumor cells. All 96 cases were diagnosed as ependymomas: 70 cases were graded similarly by all neuropathologists, while 10 cases were considered to harbor unusual features (such focal clear-cell change or tanycytic features too limited to classify tumors in these variants) or could not be graded by at least one of the reviewers and were not assigned a particular grade. In 16 other cases, one neuropathologist suggested a high grade while the other two rendered a low-grade diagnosis (Table 4).

Histological features were recorded for all 96 cases. Focal hypercellular nodules were seen in 39 cases ( $41 \%$; Figure 2a and b), while 21 tumors were considered to have diffuse hyperchromasia with pleomorphic nuclei (22\%; Figure 2c and d). Sixtysix cases had mitoses fewer than five per $10 \mathrm{HPF}$ (69\%), while 18 had between five and $10(19 \%)$ and 12 cases had mitoses greater than 10 per $10 \mathrm{HPF}$ $(12 \%)$. Necrosis was recorded in 63 cases (66\%). There was no significant difference in terms of histological features among patients enrolled in different POG protocols.

Among the 70 tumors with consensus diagnoses, 17 tumors exhibited clear vascular endothelial proliferation (Figure 3a), while in the remaining 53 cases vascular changes were not sufficient enough to be considered as bona fide vascular endothelial proliferation. Some of the 53 tumors harbored proliferating vessels that displayed a characteristic geographical arrangement, more compatible with a reactive vascular change imposed by cystic degeneration than a biologically aggressive process (Figure $3 \mathrm{~b}-\mathrm{d})$. Focal hypercellular nodules were present in 24 of 70 cases $(34.3 \%)$, diffuse hyperchromasia and pleomorphic nuclei were present in $15(21.4 \%)$, and necrosis was present in 47 cases $(67.1 \%)$. Only five

Table 4 Results of pathological review of 96 posterior fossa ependymomas by study authors

\begin{tabular}{|c|c|c|c|c|c|}
\hline Consensus & Pathologist I & Pathologist II & Pathologist III & Frequency & Percent \\
\hline \multirow[t]{6}{*}{ No (26) } & Grade II & Grade II & $\mathrm{NOS}^{\mathrm{a}}$ & 1 & 1.04 \\
\hline & Grade II & NOS $^{\mathrm{a}}$ & Grade II & 2 & 2.08 \\
\hline & Grade II & NOS & NOS & 3 & 3.13 \\
\hline & Grade III & Grade II & Grade II & 16 & 16.67 \\
\hline & Grade III & Grade II & NOS & 1 & 1.04 \\
\hline & Grade III & NOS & NOS & 3 & 3.13 \\
\hline \multirow[t]{2}{*}{ Yes (70) } & Grade II & Grade II & Grade II & 50 & 52.08 \\
\hline & Grade III & Grade III & Grade III & 20 & 20.83 \\
\hline
\end{tabular}

${ }^{\mathrm{a}}$ NOS—Cases interpreted to have unusual histological features that prevented the individual neuropathologist to accurately grade them as either Grade II or III ependymomas (see Results for a more detailed discussion). 

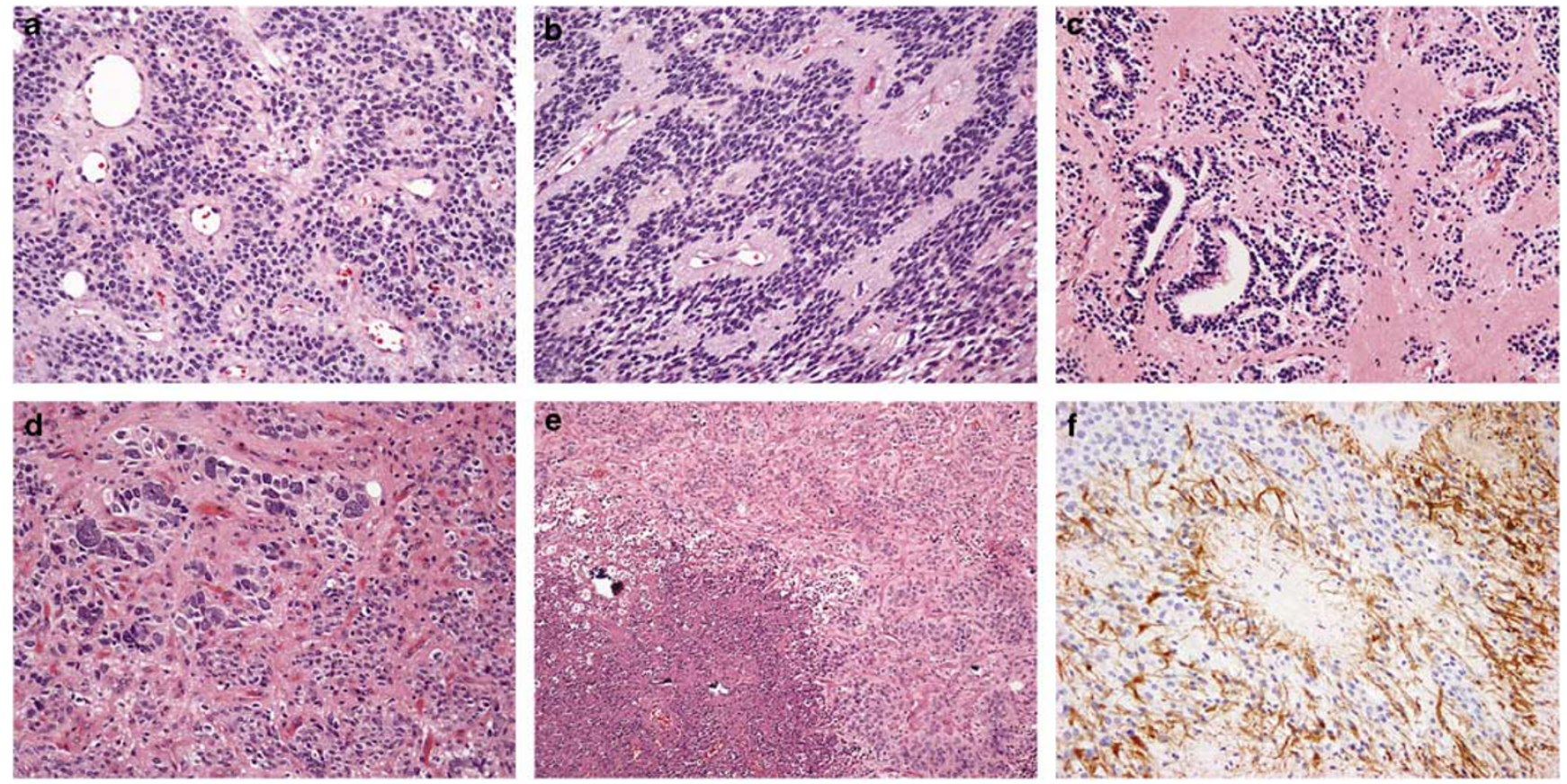

Figure 1 Histological features in Grade II ependymomas. Typical perivascular pseudorosettes representing radiating glial processes are seen two different planes of section $(\mathbf{a}, \mathbf{b})$. True rosettes were seen in a minority of cases $(\mathbf{c})$. Rare cells demonstrated bizarre morphology with nuclear hyperchromasia, but this was distinctly focal (d). Geographic necrosis without pseudopalisading was common (e). Immunohistochemical studies for glial fibrillary acidic protein (GFAP) demonstrated variable staining of tumor cells, but a distinct accentuation in the perivascular regions (f).

of these cases had classical palisading necrosis. Forty-five cases had mitoses $<5$ per $10 \mathrm{HPF}$ (64.3\%), 12 cases had >10 mitoses per $10 \mathrm{HPF}$ $(17.1 \%)$ and the mitotic rates were between five and 10 per HPF in 13 cases (18.6\%). Details of histological features in 70 cases with consensus are presented in Tables 5 and 6.

\section{Correlation of Histologic Grade or Clinical Parameters with Outcome}

There was a statistically significant difference in EFS between Grade II and III ependymomas for all pathology diagnoses (original, individual, and consensus). Log-rank $P$-values were $0.017,0.0033$, and 0.0027 for the individual pathologists, and 0.0029 for the consensus diagnoses based on 70 patients (Table 3). Figure 2 presents the EFS plot for consensus review diagnoses. Kaplan-Meier analysis for OS for Grade II and III ependymomas did not reveal significant differences even though there was a tendency for a shorter median OS for Grade III ependymomas (Figure 4).

Incorporation of one additional histological criterion (presence focal hypercellular nodules) resulted in a diminishing difference of survival probability between Grade II and III neoplasms, and the log-rank analysis did not reveal a statistical significance for EFS. Thus this criterion was not included in Table 1.

Among other parameters tested, there were significant survival differences between tumors with gross total resection and partial resection, and between patients younger and older than 3 years (Table 3). The significance was for both EFS and OS probability. Other parameters tested did not reach statistical significance in terms of EFS or OS. The impact of CSF dissemination at diagnosis was not analyzed due to very small numbers of patients with dissemination.

Multivariate Cox regression analysis was performed to assess the relative significance of age, sex, extent of resection and diagnoses as predictors of EFS. The analysis including these four factors showed that extent of resection and consensus diagnosis were strong significant predictors of increased risk of an event. The relative event risk was 3.59 for tumors with less than gross total resection and 3.58 for patients with Grade III ependymoma $(P<0.001)$.

\section{Comparison of Institutional vs Central Review Diagnosis}

The original diagnoses were WHO Grade II ependymoma in 81 patients, WHO Grade III (anaplastic) ependymoma in 12 patients, and other diagnosis in the remaining three cases. The original diagnoses agreed with the consensus diagnoses in 50 of the cases, and differed in 20 cases (agreement level $72 \%$ ). Original diagnoses were in agreement in 45 of 50 cases considered to be WHO Grade II, but agreed in only five of 20 cases considered to be WHO Grade III by the reviewers. Since we did not have specific information on the specific findings of the institu- 

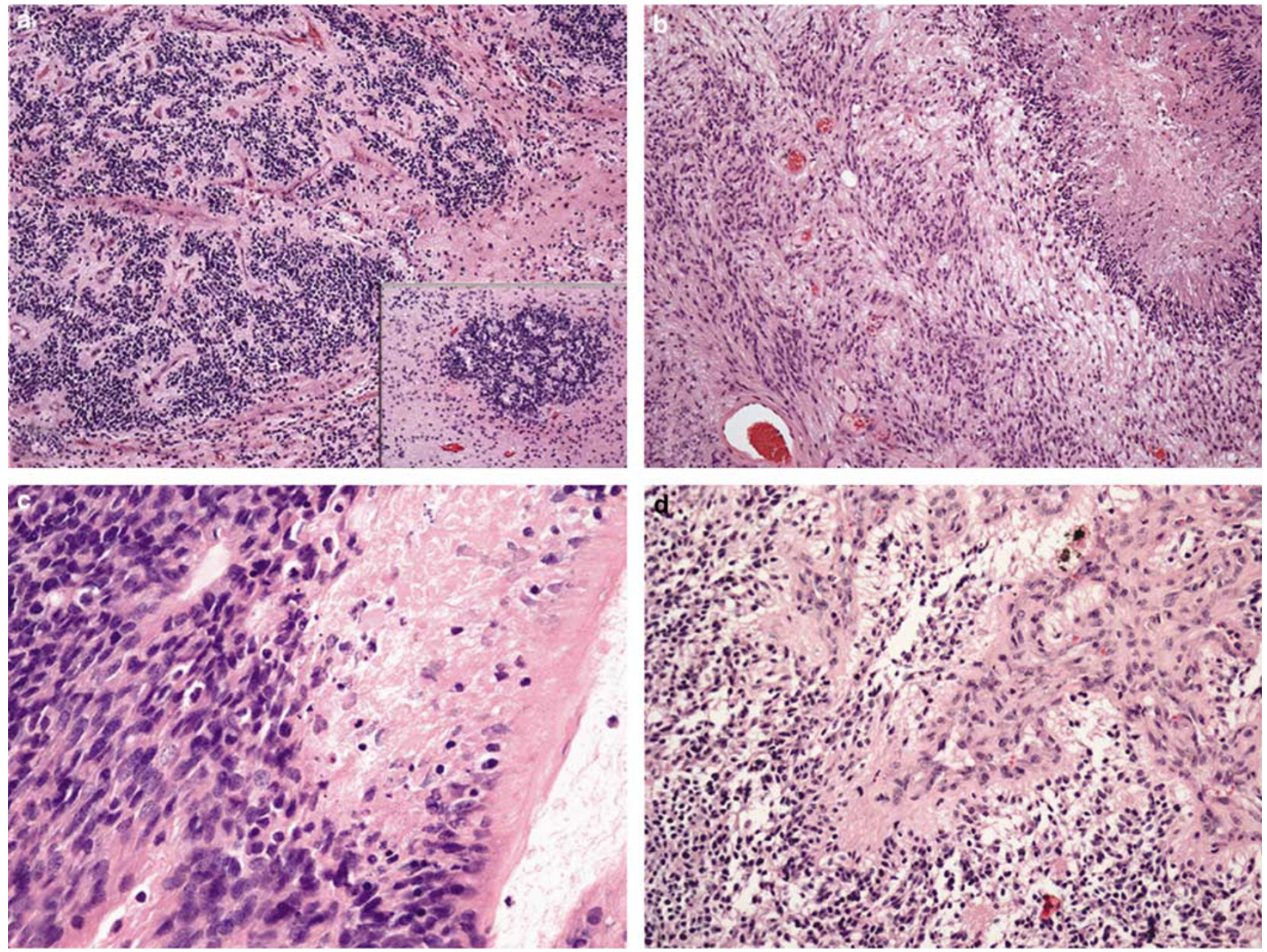

Figure 2 Atypical and anaplastic histological features. Focal hypercellular nodules were worrisome but not sufficient for a higher risk of recurrence (a). Some of these nodules were macroscopically visible, but they were often microscopic (inset). Necrosis with pseudopalisading was considered as a criterion for Grade III (b). Diffuse hypercellularity and associated nuclear pleomorphism was typical of Grade III neoplasms (c). Bona fide vascular proliferation was considered the cardinal criterion for Grade III ependymoma (d). All features except hypercellular nodules were features of Grade II ependymomas.

tional pathologists, we have analyzed the prevalence of histological features as recorded in the joint review, for tumors originally diagnosed as Grade II (81 cases) and III (12 cases). Tumors with the original diagnoses of Grade II ependymoma included 34 cases with focal hypercellular nodules, 52 cases with necrosis, 14 cases with diffuse hypercellularity and nuclear pleomorphism, and 13 cases with vascular proliferation. Among Grade II tumors 58 had $<5$ mitoses, 16 had 5-10 mitoses, and seven had $>10$ mitoses per $10 \mathrm{HPF}$. Individual histological parameters were separately correlated with tumor grade using nominal by nominal contingency coefficient and Lambda calculations. Presence of focal hypercellular nodules, necrosis, and vascular proliferation did not correlate with tumor grade, but low mitotic rate positively correlated with Grade II ependymoma $(P<0.024)$, while diffuse hypercellularity with nuclear pleomorphism positively correlated with Grade III ependymoma
$(P<0.004)$. Survival analysis based on the original diagnoses demonstrated a significant difference between Grade II and III ependymomas in terms of EFS. In addition, the difference was significant in terms of OS.

\section{Literature Review}

We identified 32 studies conducted between 1990 and 2005 that conformed to the inclusion/exclusion criteria (see Materials and methods). ${ }^{3-7,9-35}$ These studies covered various time frames ranging from 2 to 45 years, and included patients treated during a 52-year period (1951-2003). There were a total of 1444 recorded cases, with eight studies including patients between ages 15 and 20 (Table 2, see age range column). A number of groups had analyzed the same patient population in more than one report with a slightly different focus, or reanalyzed 

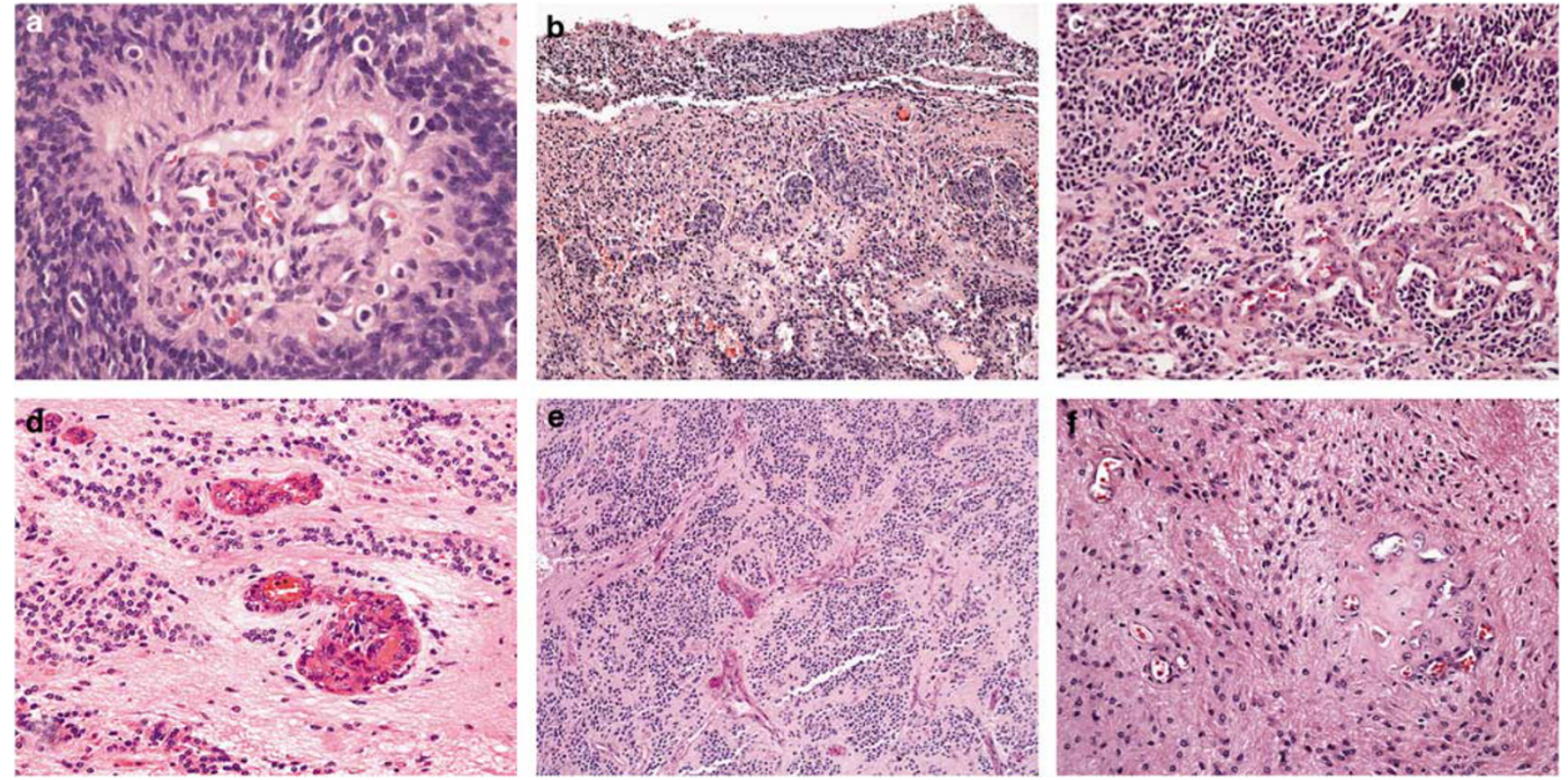

Figure 3 Spectrum of vascular changes in ependymomas. (a) High-power magnification of vascular endothelial proliferation showing prominent layering of endothelial cells, often dissociated from lumen formation. A reticulin staining of this particular section revealed that the proliferation of endothelial cells was not in relation to the vascular lumen (not shown). Other types of vascular changes not considered as bona fide vascular endothelial proliferation included linear proliferations of tufted vessels often related to cystic/ degenerative changes (b, c), small cluster of vessels with equivocal increase in endothelial cells (d), prominent but delicate vascular network (e), and hyalinized vessels (f).

Table 5 Histopathological findings in 70 patients with consensus diagnoses as recorded on central review

\begin{tabular}{|c|c|c|c|c|c|c|c|}
\hline \multirow{2}{*}{$\begin{array}{l}\text { Vascular endothelial } \\
\text { proliferation }\end{array}$} & \multirow{2}{*}{$\begin{array}{l}\text { Focal hypercellular } \\
\text { nodules }\end{array}$} & \multirow[t]{2}{*}{ Necrosis } & \multirow{2}{*}{$\begin{array}{l}\text { Hypercellularity and } \\
\text { nuclear pleomorphism }\end{array}$} & \multirow[t]{2}{*}{ Mitoses } & \multicolumn{2}{|c|}{ Consensus grade } & \multirow[t]{2}{*}{ Total } \\
\hline & & & & & Grade II & Grade III & \\
\hline \multirow[t]{10}{*}{ No (53) } & No (40) & No (15) & No & $<5$ & 15 & & 15 \\
\hline & & & No (18) & $5-10$ & 1 & & 1 \\
\hline & & & & $<5$ & 17 & & 17 \\
\hline & & Yes (25) & Yes (7) & $5-10$ & 1 & & 1 \\
\hline & & & & $<5$ & 3 & & 3 \\
\hline & & & & $>10$ & & 3 & 3 \\
\hline & Yes (13) & No (5) & No & $5-10$ & 2 & & 2 \\
\hline & & & & $<5$ & 3 & & 3 \\
\hline & & Yes (8) & No & $5-10$ & 1 & & 1 \\
\hline & & & & $<5$ & 7 & & 7 \\
\hline \multirow[t]{9}{*}{ Yes (17) } & No (6) & No (1) & Yes & $>10$ & & 1 & 1 \\
\hline & & Yes (5) & Yes & $5-10$ & & 3 & 3 \\
\hline & & & & $>10$ & & 2 & 2 \\
\hline & Yes (11) & No (2) & No & $5-10$ & & 1 & 1 \\
\hline & & & & $>10$ & & 1 & 1 \\
\hline & & Yes (9) & No (7) & $5-10$ & & 3 & 3 \\
\hline & & & & $>10$ & & 4 & 4 \\
\hline & & & Yes (2) & $5-10$ & & 1 & 1 \\
\hline & & & & $>10$ & & 1 & 1 \\
\hline
\end{tabular}

Numbers in bold identify anaplastic (Grade III) ependymomas.

subgroups for a different purpose..$^{5,7,9,11,12,17,28,35}$ Therefore, we presume that the actual number of patients was fewer, but an accurate count was not possible since data were lacking on which patients were reanalyzed in different studies.

The median age at diagnosis for all patients was 4.2 years (mean 6.1 years). One study exclusively included children 3 years or younger. Male to female ratio varied considerably among studies from 0.7 to 1.7 , and the mean ratio was 1.2 , suggesting an equal gender distribution for this neoplasm. The tumors were in the posterior fossa in 939 patients and were supratentorial in 446 patients. Two patients in one study were recorded to have both infra- and 
Table 6 Multivariate analysis of EFS in 70 posterior fossa ependymomas with consensus diagnoses

\begin{tabular}{|c|c|c|c|c|}
\hline Factor & Category & Relative risk & $95 \%$ Confidence intervals & $\mathrm{P}$-value \\
\hline Extent of resection & $\begin{array}{l}\text { Gross total } \\
<\text { Total }\end{array}$ & $\begin{array}{l}1.00 \\
3.59\end{array}$ & $1.9-6.8$ & $<\mathbf{0 . 0 0 1}$ \\
\hline Consensus review diagnosis & $\begin{array}{l}\text { Grade II } \\
\text { Grade III }\end{array}$ & $\begin{array}{l}1.00 \\
3.58\end{array}$ & $1.8-7.0$ & $<0.001$ \\
\hline Age & $\begin{array}{l}<3 \text { years } \\
>3 \text { years }\end{array}$ & $\begin{array}{l}1.00 \\
0.57\end{array}$ & $0.3-1.05$ & 0.07 \\
\hline Sex & $\begin{array}{l}\text { Male } \\
\text { Female }\end{array}$ & $\begin{array}{l}1.00 \\
0.80\end{array}$ & $0.4-1.5$ & 0.48 \\
\hline
\end{tabular}

EFS, event-free survival.

Statistically significant values are presented in bold.
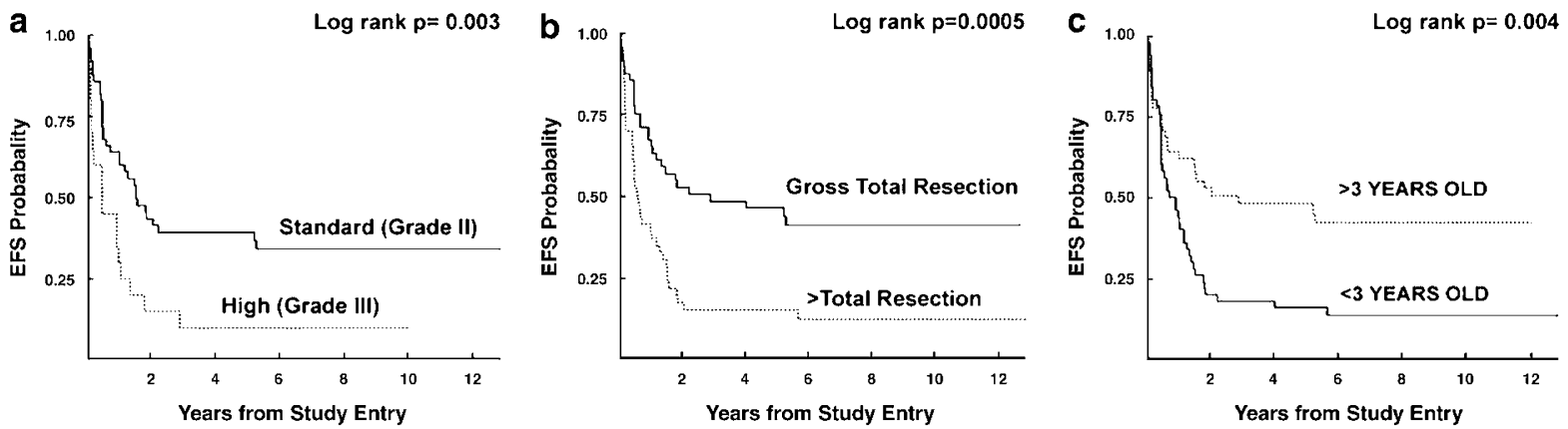

Figure 4 Kaplan-Meier survival curves for the impact of (a) grade, (b) extent of surgery, and (c) age on EFS.

supratentorial tumors at presentation. The location was not specified in one study of 48 patients. ${ }^{22}$ Most of the studies reported using WHO criteria for histological grading of tumors. Among studies where histological grading information was reported, there were 638 WHO Grade II tumors and 543 WHO Grade III tumors. In the remaining 263 tumors, the grade was not specified as either II or III.

\section{Influence of extent of surgery}

All but one study reported extent of initial resection, which was gross total in 741 , subtotal in 604 , and biopsy in 25 cases. In the remaining 74 cases, extent of resection could not be determined. Extent of resection was reported as an independent prognostic factor in 21 of the studies. In nine studies, the sample size was either too small or too few patients had reached end points to determine statistical significance. The remaining two studies reported, but did not analyze the significance of extent of resection, which was not within the scope of their analysis. Extent of surgery was significant for both EFS and OS in 11 studies, for OS in six, and for EFS or progression-free survival in four studies. The significance of extent of resection was demonstrated in all studies where the sample size was sufficient, and where both EFS and OSs were reported. In summary, extent of resection was a critical independent prognostic indicator for both EFS and OS in pediatric ependymomas.
Influence of histological grade

Among 32 studies, 24 reported using the WHO grading scheme, or at least utilized the broad principles of the 1993 or 2000 WHO schemes. Eight others either did not use WHO grading scheme, did not mention the method of histological grading, or did not provide the histological grade of tumors. Histological grade was calculated as a prognostically significant factor in nine of the 32 studies analyzed..$^{4,10,12,20,25,30,32-34}$ One additional study found necrosis as a significant prognostic factor, but this study did not find anaplastic histology to be prognostically significant. ${ }^{7}$ Eight of nine studies found that histological grade was a significant prognostic factor in terms of EFS or progressionfree survival. In addition, five of the studies found a statistically significant negative impact of histological grade on OS.

Twelve studies found no impact of histological features or histological grade (when recorded) on survival. Three of these studies did not use WHO guidelines and one did not specify how grading was performed. One study provided no grading information and only provided an analysis of Ki-67 labeling on outcome. One study included only children younger than 3 years and two others analyzed survival with individual histological parameters. Two other studies failed to find age or extent of resection as significant prognostic factors in addition to histological grade. Two others found extent of resection as a significant prognostic variable, but did not find age or histologic grade to be significant. 
The remaining 11 studies had either too small numbers to analyze significance, did not provide a histological grade, or used only patients with anaplastic (Grade III) ependymomas (two studies). Most of these studies had too few cases for statistical analysis once the cohorts were segregated by grade, extent of resection, and age group.

The ratio of Grade II and III ependymomas varied in each study. Many studies had Grade II ependymomas that were up to two to three times more frequent than Grade III tumors. ${ }^{3,4,6,9,11,14,15,17,20,22,24,25,30}$ In some studies this ratio was almost equal, ${ }^{13,19,33-35}$ while in others there were far more Grade III than Grade II ependymomas. ${ }^{12,26,28,29,31,32}$ In one study of ependymomas in children under 5 years of age, the authors reported eight Grade II and 60 Grade III ependymomas. ${ }^{26}$ However, since none of the studies were apparently designed to provide prevalence of demographic or pathological features in a catchment area, these variations reflected a selection bias in location, age, or available material.

\section{Influence of age at diagnosis}

Stratification of patients based on age varied among the studies. While nine of the studies included patients older than 15 years (but younger than 20 years), only one study analyzed children under the age of 3. Among 18 studies where the effect of age on survival was analyzed, 12 studies reported age at diagnosis as an independent prognostic factor. ${ }^{4,7,9-11,13,20,22,24,25,33,34}$ Six of these studies considered the cut-off for age groups as 3 years, two as 4 years, and one as 5 years of age. In one study, age was calculated as a continuous variable, and in another study of children younger than 3 years, the cut-off between two age groups was 2 years of age (24 months). In six studies, age was not a significant prognostic factor and in the remaining 14 studies, age was either not analyzed as a prognostic variable, or the sample size was too small to determine significance.

\section{Influence on tumor location}

Among the 32 studies reviewed, three involved only supratentorial tumors and two analyzed infratentorial tumors. In only one study, tumor location was not recorded. ${ }^{22}$ Tumor location was reported to significantly affect prognosis in six studies. ${ }^{17-19,24,26,31}$ In all the studies with positive findings, posterior fossa tumors had a far less favorable outcome, but only three of these studies provided a multivariate analysis in which location still remained a significant prognostic factor ${ }^{24,26,31} \mathrm{In}$ the remaining 19 studies, location was either not analyzed as a prognostic factor or was found not to influence survival.

\section{Influence of other factors}

Survival was positively influenced by administration of radiotherapy in seven studies, and two studies reported improved survival with chemotherapy regimens. In 18 studies, the role of radiation therapy on outcome was not analyzed, and in the remaining seven reports, radiation treatment did not significantly impact survival. Comparison of treatment modalities among studies was extremely difficult due to different patient populations, age groups, types and modes of treatments, and the number of studies that did not specifically evaluate the effects of treatment. Therefore, no further analysis of treatment modalities was attempted. One study reported a significant relationship between OS as well as progression-free survival and duration of symptoms before surgery. ${ }^{7}$ Another study reported significance of metastatic (M) stage at diagnosis negatively affected survival. ${ }^{34}$ The only study that analyzed genomic imbalances detected a negative effect of chromosomal imbalance on OS. ${ }^{29}$

\section{Discussion}

There are many challenges inherent in retrospective, single-institution studies of rare neoplasms such as pediatric ependymomas. First, the studies typically involve decades of clinical practice to accumulate sufficient numbers. The second group of problems relate to the substantial variations in clinical characteristics such as age, tumor location, or histological subtype, while a third group relates to variations in diagnostic modalities (CT vs MRI), surgical approach, adjuvant treatment, and pathological interpretation. These variables result in limited number of patients with uniform set of attributes, and often fail to provide sufficient statistical power to interpret negative results.

Despite such challenges, the results of recent studies on ependymomas leave little doubt on the importance of surgical resection regardless of age group, tumor location, or histological grade. Our findings in 96 patients from POG studies as well as the overwhelming majority of the reported studies provide convincing data for this suggestion. Like many others, we believe that an attempt for gross total resection provides the best survival advantage for children with ependymomas.

There is also strong evidence that younger children have a higher risk of recurrence or adverse outcome, and the cut-off age for risk stratification seems to be 3 years, even though studies that have chosen 4 or 5 years as cut-off limit were also successful in demonstrating a survival disadvantage for young age., ${ }^{4,20,24}$ One study suggested that children a year younger than this age limit (ages 0-24 months) could have even higher risk than children in their third year. ${ }^{36}$ Small number of welldesigned studies with sufficient power demonstrated that age was not dependent on extent of surgical resection. ${ }^{4,25,33}$ Even though a specific biological reason eludes all the studies, and the rationale for this choice is not clear, 3 years of age 
seems to be the most appropriate limit for stratification. This fact provided our rationale for using this age limit in our study. The composition of patients in our study was slightly biased toward younger children due to some of the POG protocols that specifically targeted children younger than 3 years of age. This gave us a chance to accrue sufficient numbers of children above and below this age limit to provide a valid analysis. In many other studies, limited number of patients younger than 3 years might have been the reason for not finding age as an independent prognostic factor. ${ }^{28}$ Another remote possibility may be that risk of young age could be more relevant for tumors in a particular location, ${ }^{31}$ even though there are studies that reported age as a prognostic variable independent of tumor location..$^{425,34}$

It is possible to suggest that tumors in the very young may pose unique challenges in clinical management or they may possess more aggressive biological properties. Circumstantial evidence for these observations is provided by Duffner et al. ${ }^{22}$ There may also be technical, therapeutic, or genetic differences between the very young and older children and this issue still requires a more detailed analysis.

Our main goal was to demonstrate the relationship between histological grade and outcome. Our results demonstrated a clear difference of EFS between Grade II and III neoplasms using both uni- and multivariate analyses. This was true for initial institutional diagnosis, individual neuropathologists' diagnoses as well as for consensus diagnosis. We have opted to utilize the criteria outlined by the current WHO classification scheme and studies that have used similar to identical criteria have also identified the importance of histological grading as an independent prognostic indicator in wider age groups and varying tumor locations. ${ }^{37,38}$ While our results are relevant to typical ependymomas located in the posterior fossa in the pediatric population (15 years or younger), a number of other studies in more heterogeneous patient populations reported similar findings in adults or supratentorial ependymomas. ${ }^{4,25}$

In this study, we followed the basic principles of the current WHO classification scheme for grading ependymomas. While this scheme provided the fundamental elements of grading, certain criteria needed to be more specifically defined. It is tempting to conclude, especially for neuro-oncologists, that the mere statement of using WHO criteria implies a standard, uniform, and reproducible scheme by the pathologist. Unfortunately, this assumption often fails once specific criteria to grade ependymomas are closely scrutinized. Most studies select arbitrary criteria for the threshold values of histological features used to segregate histological grades. Similarly, we adopted a set of arbitrary parameters, such as in frequency of mitoses, which were primarily developed following our review of the literature and our collective experience. Thus, while the selection of our criteria is an attempt to validate WHO classification, the selection of specific cut-off values were obtained from a critical review of the literature and were considered evidence-based rather than a priori.

Briefly, a typical (WHO Grade II) ependymoma had prominent perivascular pseudorosettes with relatively uniform nuclei, less than five mitotic figures per 10 high-power magnification $(\times 40)$ field, and lacked endothelial vascular proliferation or palisading necrosis. Tumors with this description were uniformly recorded as Grade II by almost every pathologist.

We allowed the presence of focal nuclear pleomorphism, focal increase in mitotic figures (5-10 mitotic figures per 10 high-power magnification), focal hypercellular nodules, and necrosis without palisading within Grade II category. Vascular proliferation of the type seen in cystic change or degeneration was also allowed. The tumors with some of these 'atypical' features often received different grades and sometimes remained excluded from the consensus due to 'unusual' character. We also noted in our literature review that use of the WHO grading scheme by different authors often differed in terms of these features. In particular, number of mitotic figures for each category often varied, and different types of necrosis and vascular proliferations were ignored. The latter, in our opinion, is one misleading feature that has been a well-recognized but less published area of contention.

Anaplastic or high-risk (WHO Grade III) ependymomas typically contained bona fide vascular endothelial proliferation, diffuse hyperchromasia with nuclear pleomorphism, marked hypercellularity, mitotic rate greater than 10 per high-power field, and/or palisading necrosis. Tumors with mitotic rates between five and 10 high-power fields were considered in this category only if they had additional criteria such as bona fide vascular proliferation. While tumors fulfilling more than a few of these criteria were easily interpreted by all pathologists as being anaplastic, one of the main discrepancies between original and central review diagnoses was the identification of features necessary to consider an ependymoma as anaplastic. Our findings also imply that inclusion of 'focal hypercellular nodules' was not helpful in differentiating two grades (see Figure 5a), and was therefore not included in the criteria presented in Table 1 . The use of this criterion in the modified grading scheme was to demonstrate the fact that inclusion of certain features is likely to render that particular scheme insignificant as a prognostic indicator.

After review of the literature relevant to pediatric posterior fossa ependymomas, it seems pretty clear that some of the differences in the results of the studies can be attributed to variations in pathological interpretation and applied criteria. According to 

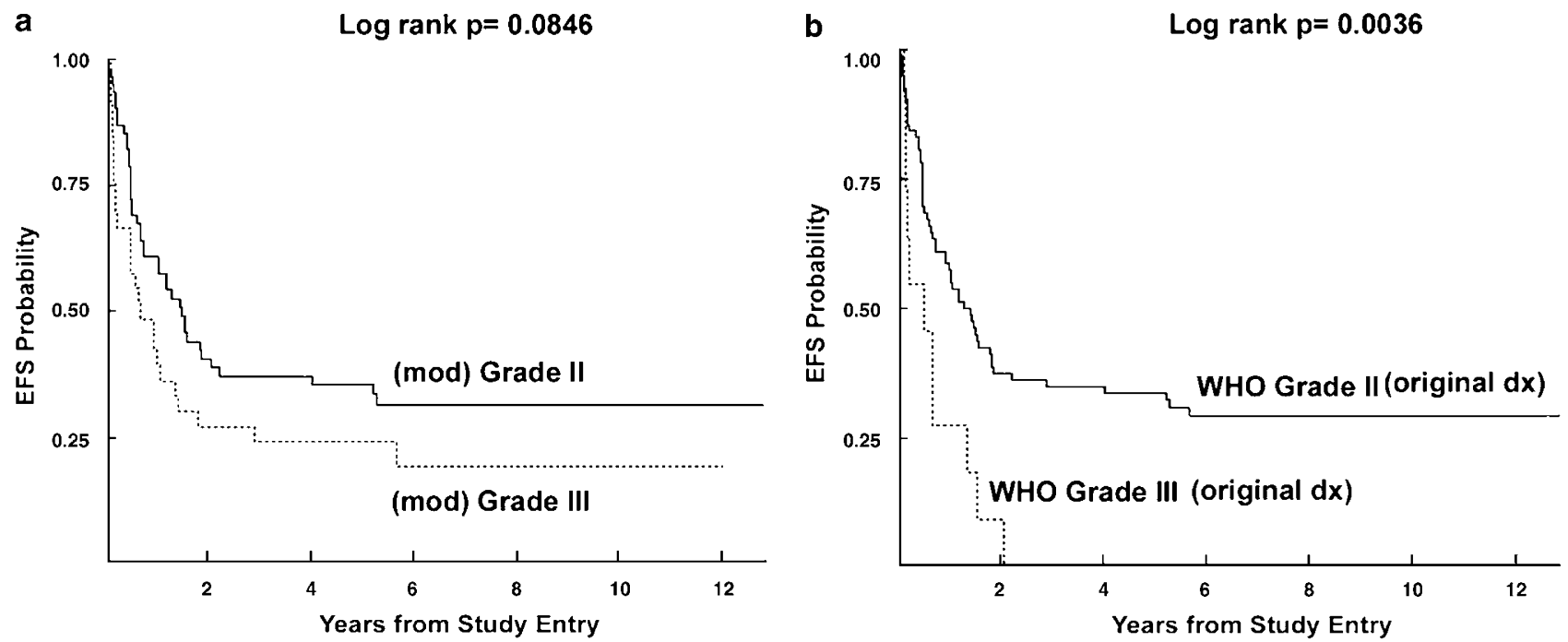

Figure 5 Kaplan-Meier survival curves for the impact of two additional schemes. (a) The modified grading scheme obtained by including focal hypercellular nodules, which resulted in loss of significance in survival difference. (b) The survival probability based on original diagnosis on EFS, which reveals a similarly significant EFS difference between Grade II and III ependymomas.

us, a statement that suggests the use of WHO classification scheme does not necessarily imply a standard and reproducible set of criteria. Similar to the progress in grading meningiomas in adults, a more reproducible grading scheme may have to wait for more precise definitions of criteria, and their uniform application. ${ }^{39}$ Even then, and only through further refinement, morphological analysis is likely to provide a more reproducible and reliable measure of biological aggressiveness. It is also important to mention that grading schemes other than the WHO classification could also be used for determining histological grade or 'anaplasia' and may yield similarly significant results. Nevertheless, a closer look at most studies that have identified histological grade as significant prognostic factor reveals that the criteria utilized in the overwhelming majority of these studies are quite similar.

While there is substantial merit in the critical review of a surgical specimen by an expert, this virtue may sometimes be all too exaggerated. Our analysis of initial pathology diagnosis in 96 patients provided a similar significance of findings for EFS as well as OS when compared with any of the expert diagnoses. This has clearly not been the case for many other neoplasms or diseases. Nevertheless, original diagnoses in this particular study are just as valid interpretations in predicting outcome. This conclusion was reached in a recent workshop of the Brain Tumor Epidemiology Consortium of neuropathologists and epidemiologists (http://rarediseases. info.nih.gov/html/workshops/brain20050926.html). A consensus in this workshop pointed to a similar conclusion with regards to histological grading of ependymomas, depending on location. Our findings point to the suggestion that posterior fossa ependymomas can also be considered within the category of tumors for which a central review is not as critical. It is tempting to assume that direct interaction with the clinical colleagues in this group of patients could provide an advantage over central reviewers who typically perform their analysis in 'blinded' manner.

In summary, we believe that there are sufficient data to consider histological grade in addition to young age and extent of resection, as a critical parameter that can influence recurrence of a pediatric posterior fossa ependymoma. While this can be said for age and extent of resection on OS, our data do not provide conclusive evidence that histological grade is a prognostically significant factor for OS. In future studies, it will be important to determine whether there are additional prognostic factors that can help better stratify patients to predict OS.

\section{References}

1 Wiestler OD, Schiffer D, Coons S, et al. Ependymoma. In: Kleihues P, Cavenee WK (eds). Pathology and Genetics of Tumours of the Nervous System World Health Organization Classification of Tumours. IARC Press: Lyon, 2000, pp 72-76.

2 Bouffet E, Perilongo G, Canete A, et al. Intracranial ependymomas in children: a critical review of prognostic factors and a plea for cooperation. Med Pediatr Oncol 1998;30:319-329.

3 Robertson PL, Zeltzer PM, Boyett JM, et al. Survival and prognostic factors following radiation therapy and chemotherapy for ependymomas in children: a report of the Children's Cancer Group. J Neurosurg 1998;88: 695-703.

4 Figarella-Branger D, Civatte M, Bouvier-Labit C, et al. Prognostic factors in intracranial ependymomas in children. J Neurosurg 2000;93:605-613.

5 Gerszten PC, Pollack IF, Martinez AJ, et al. Intracranial ependymomas of childhood. Lack of correlation of 
histopathology and clinical outcome. Pathol Res Pract 1996;192:515-522.

6 Foreman NK, Love S, Thorne R. Intracranial ependymomas: analysis of prognostic factors in a population-based series. Pediatr Neurosurg 1996;24:119-125.

7 Pollack IF, Gerszten PC, Martinez AJ, et al. Intracranial ependymomas of childhood: long-term outcome and prognostic factors. Neurosurgery 1995;37:655-666.

8 Kleihues P, Louis DN, Scheithauer BW, et al. The WHO classification of tumors of the nervous system. J Neuropathol Exp Neurol 2002;61:215-225.

9 Goldwein JW, Leahy JM, Packer RJ, et al. Intracranial ependymomas in children. Int J Radiat Oncol Biol Phys 1990;19:1497-1502.

10 Nazar GB, Hoffman HJ, Becker LE, et al. Infratentorial ependymomas in childhood: prognostic factors and treatment. J Neurosurg 1990;72:408-417.

11 Sutton LN, Goldwein J, Perilongo G, et al. Prognostic factors in childhood ependymomas. Pediatr Neurosurg 1990;16:57-65.

12 Ernestus RI, Wilcke O, Schroder R. Supratentorial ependymomas in childhood: clinicopathological findings and prognosis. Acta Neurochir (Wien) 1991;111: 96-102.

13 Chiu JK, Woo SY, Ater J, et al. Intracranial ependymoma in children: analysis of prognostic factors. J Neurooncol 1992;13:283-290.

14 Palma L, Celli P, Cantore G. Supratentorial ependymomas of the first two decades of life. Long-term followup of 20 cases (including two subependymomas). Neurosurgery 1993;32:169-175.

15 Rousseau P, Habrand JL, Sarrazin D, et al. Treatment of intracranial ependymomas of children: review of a 15-year experience. Int J Radiat Oncol Biol Phys 1994; 28:381-386.

16 Jayawickreme DP, Hayward RD, Harkness WF. Intracranial ependymomas in childhood: a report of 24 cases followed for 5 years. Childs Nerv Syst 1995;11: 409-413.

17 Ernestus RI, Schroder R, Stutzer H, et al. Prognostic relevance of localization and grading in intracranial ependymomas of childhood. Childs Nerv Syst 1996; 12:522-526.

18 Merchant TE, Haida T, Wang MH, et al. Anaplastic ependymoma: treatment of pediatric patients with or without craniospinal radiation therapy. J Neurosurg 1997;86:943-949.

19 Needle MN, Goldwein JW, Grass J, et al. Adjuvant chemotherapy for the treatment of intracranial ependymoma of childhood. Cancer 1997;80:341-347.

20 Perilongo G, Massimino M, Sotti G, et al. Analyses of prognostic factors in a retrospective review of 92 children with ependymoma: Italian Pediatric Neurooncology Group. Med Pediatr Oncol 1997;29:79-85.

21 Bennetto L, Foreman N, Harding B, et al. Ki-67 immunolabelling index is a prognostic indicator in childhood posterior fossa ependymomas. Neuropathol Appl Neurobiol 1998;24:434-440.

22 Duffner PK, Krischer JP, Sanford RA, et al. Prognostic factors in infants and very young children with intracranial ependymomas. Pediatr Neurosurg 1998; 28:215-222.
23 Fouladi M, Baruchel S, Chan H, et al. Use of adjuvant ICE chemotherapy in the treatment of anaplastic ependymomas. Childs Nerv Syst 1998;14:590-595.

24 Sala F, Talacchi A, Mazza C, et al. Prognostic factors in childhood intracranial ependymomas: the role of age and tumor location. Pediatr Neurosurg 1998;28: 135-142.

25 Horn B, Heideman R, Geyer R, et al. A multiinstitutional retrospective study of intracranial ependymoma in children: identification of risk factors. J Pediatr Hematol Oncol 1999;21:203-211.

26 Grill J, Le Deley MC, Gambarelli D, et al. Postoperative chemotherapy without irradiation for ependymoma in children under 5 years of age: a multicenter trial of the French Society of Pediatric Oncology. J Clin Oncol 2001;19:1288-1296.

27 Helseth E, Due-Tonnessen B, Lote K, et al. Ependymoma in children and young adults (0-19 years): report of 25 consecutive cases. Childs Nerv Syst 2001;17:24-30.

28 Vinchon M, Soto-Ares G, Riffaud L, et al. Supratentorial ependymoma in children. Pediatr Neurosurg 2001; 34:77-87.

29 Dyer S, Prebble E, Davison V, et al. Genomic imbalances in pediatric intracranial ependymomas define clinically relevant groups. Am J Pathol 2002 ;161:2133-2141.

30 Merchant TE, Jenkins JJ, Burger PC, et al. Influence of tumor grade on time to progression after irradiation for localized ependymoma in children. Int J Radiat Oncol Biol Phys 2002;53:52-57.

31 van Veelen-Vincent ML, Pierre-Kahn A, Kalifa C, et al. Ependymoma in childhood: prognostic factors, extent of surgery, and adjuvant therapy. J Neurosurg 2002; 97:827-835.

32 Zamecnik J, Snuderl M, Eckschlager T, et al. Pediatric intracranial ependymomas: prognostic relevance of histological, immunohistochemical, and flow cytometric factors. Mod Pathol 2003;16:980-991.

33 Jaing TH, Wang HS, Tsay PK, et al. Multivariate analysis of clinical prognostic factors in children with intracranial ependymomas. J Neurooncol 2004;68:255-261.

34 Agaoglu FY, Ayan I, Dizdar Y, et al. Ependymal tumors in childhood. Pediatr Blood Cancer 2005;45:298-303.

35 Vinchon M, Leblond P, Noudel R, et al. Intracranial ependymomas in childhood: recurrence, reoperation, and outcome. Childs Nerv Syst 2005;21:221-226.

36 Duffner PK, Horowitz ME, Krischer JP, et al. The treatment of malignant brain tumors in infants and very young children: an update of the Pediatric Oncology Group experience. Neurooncol 1999;1:152-161.

37 Ho DM, Hsu CY, Wong TT, et al. A clinicopathologic study of 81 patients with ependymomas and proposal of diagnostic criteria for anaplastic ependymoma. J Neurooncol 2001;54:77-85.

38 Korshunov A, Golanov A, Sycheva R, et al. The histologic grade is a main prognostic factor for patients with intracranial ependymomas treated in the microneurosurgical era: an analysis of 258 patients. Cancer 2004;100:1230-1237.

39 Perry A, Stafford SL, Scheithauer BW, et al. Meningioma grading: an analysis of histologic parameters. Am J Surg Pathol 1997;21:1455-1465. 